\title{
CONSTRUCTIONS DU CENTRE DE COURBURE DE LA COURBE, LIEU DES POINTS DONT LES DISTANCES A DEUX COURBES DONNÉES SONT DANS UN RAPPORT CONSTANT
}

\section{PAR A. MANNHEIM.}

CAPITAINE D'ARTILLERIE.

LESME. D'un point quelconque a $\left(\mathrm{fig}^{\mathrm{a}} \cdot \mathrm{1}^{\mathrm{a}}\right.$.) de la courbe $\mathrm{A}$, on abaisse la normale ab sur la courbe $\mathrm{B}$; aux points $\mathrm{a}, \mathrm{b}$ on mène les tangentes $\mathrm{al}$, bl aux courbes $\mathrm{A}, \mathrm{B}$; lorsque a parcourt $\mathbf{A}$, le point $\mathbf{l}$ décrit un lieu dont on demande de construire la tangente $\mathbf{L}$.

Soient $\mathbf{A}^{\prime}$ et $\mathbf{B}^{\prime}$ des courbes symétriques à $\mathbf{A}$ el à $\mathbf{B}$ par rapport à la tangente al; fesons rouler $\mathbf{A}^{\prime}$ sur $\mathbf{A}$, soit $\mathbf{D}$ la courbe enveloppe de $\mathbf{B}^{\prime}$ entrainée dans ce mouvement; $D$ touche $B^{\prime}$ au point $e$ pied de la normale abaissée du point $a$ sur $B^{\prime}$; cc point est le symétrique de $b$, par suite la tangente en $e$ à la courbe $D$ passe par $l$, en outre les tangentes $l e$ el $l b$ sont égales. D'apris cela, en remplacant les courbes $\mathrm{B}$ el $\mathrm{D}$ par leurs cercles osculateurs en $b$ et en $e$, lo point $l$ décrit une droite, axe radical de ces circonférences. Il nous reste à construire cet axe radical qui n'est autre chose que la tangente demandée.

Cet axe passe par le point $l$ et doit être perpendiculairc à la ligne des centres des cercles osculateurs; on connait le centre de courbure $f$ de $B$, cherchons celui de D correspondant au point $e$.

Le centre de courbure de $\mathrm{D}$, correspondant au point $e$, n'est autre que le centre de courbure de la courbe décrite par $g$, centre de courbure de $B^{\prime}$; en appliquant la construction de Savary, comme l'indique la figure, on trouve le point $h$.

Nous allons modifier la construction de ce point. D'après la formule de Savary l'on a:

$$
\left(\frac{1}{a h}+\frac{1}{g a}\right) \cos . o a h=\frac{2}{a o},
$$

d'un autre côté, en menant des points $h$ et $q$ les parallèles $h m$ et $q p$ à la tangente $a l$, l'on obtient quatre points $a, m, p, f$ qui forment une dirision harmonique et l'on a:

$$
\frac{1}{a m}+\frac{1}{a f}=\frac{2}{a p}
$$

en comparant celte relation avec la précédente, et remarquant quc $a m=a h, g a=a f$, on conclut que le point $p$ est la projection du centre de courbure $o$ sur la droite $a f$. D'après cela, il suffit d'abaisser du point o la perpendiculaire op sur fa, du 
point $p$, la perpendiculaire $p q$ sur $a o$, et l'on obtiendra, en joignant le point $f$ au point $q$, une ligne $f q$ qui passe par le point $h$.

L'axe radical cherché, qui n'est autre que la tangente demandée, est la perpendiculaire $L$ abaissée du point $l$ sur $f q$.

Réciproquement connaissant celte tangente, on peut, à l'aide de la construction inverse, déterminer le centre de courbure de l'une des courbes, l'autre centre étant supposé connu.

$2^{\text {me }}$. Solution. Remplaçons la courbe A par une conique ayant pour foyer le point $f$, pour centre de courbure $o$ et passant en $a$; remplaçons en $b$ la courbe $\mathbf{B}$ par son cercle osculateur.

Cette conique et ce cercle sont deux courbes homologiques ayant $f$ pour centre d'homologie; le point $l$ décrit alors un axe d'homologie (Prop. proj. des fig. $n$. 459). Cet axe étant perpendiculaire à la ligne des foyers de la conique, il faut pour le construire, chercher cetle dernière droite.

Pour cela, on abaisse du centre de courbure o la perpendiculaire op sur $f a$, du point $p$ la perpendiculaire $p q$ sur $a o$, la ligne $f q$ passe par le second foyer de la conique; la perpendiculaire $L$ abaissée du point $l$ sur $f q$ est la droite cherchée. On retrouve ainsi la première construction.

On arrive à une autre construction de la manière suivante: l'axe d'homologie que nous cherchons étant parallèle à la directrice de la conique, il suffit de chercher cette droite. Le point $l$, intersection de la tangente al et de la ligne $f l^{\prime}$ perpendiculaire à $f a$, est un point de cette directrice; au point $l^{\prime}$ on élìve à $l^{\prime} a$ la perpendiculaire $l^{\prime} t$, cette droite rencontre of au point $t$; la ligne $t a$ est perpendiculaire à la directrice $L^{\prime}$

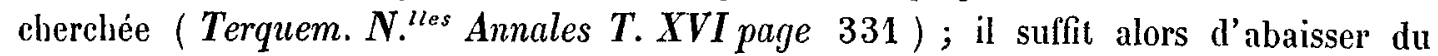
point $l$ la perpendiculaire $L$ sur $t a$ pour avoir la langente demandée.

Reanaques. 1: Nous venons de voir qu'il suffisait de chercher $L^{\prime}$; nous avons déjà donné une construction de cette droite dans les Nouvelles Annales à l'article que nous venons de citer.

Voici la question résolue au n: 21 de cet article :

"Soit $b f c$ un triangle rectangle mobile et variable dont le sommet de l'angle " droit est fixe en $f$ et dont l'hypoténuse $b c$ est tangente à une circonférence donnée " o, le point $c$ est le point de contact mobile: on demande la normale au lieu dé" crit par le point $b$."

On peut trouver un grand nombre de constructions de la tangente au lieu décrit par le point $b$ et par suite de $L$ ', il suffit pour cela de transformer, à l'aide de la théoric des polaires réciproques, les constructions connues du centre de courbure des coniques, la directrice étant une circonférence décrite de l'un des foyers de cette conique comme centre. 
Réciproquement une construction de la tangente au lieu décrit par le point $b$ conduit, à l'aide de la théorie des polaires réciproques, à une construction du centre de courbure des coniques.

$2^{\circ}$. Le lemme que nous avons résolu plus haut est un cas particulier de la question suivante:

Etant donnés deux courbes quelconques $\mathrm{A}, \mathrm{B}$ et un point $\mathrm{f}$ arbitraire dans le plan de ces courbes, de ce point, on mène une transversale quelconque qui coupe, $\mathrm{A}$ au point a et $\mathrm{B}$ au point $\mathrm{b}$; de ces points, on mène aux courbes $\mathrm{A}$ et $\mathrm{B}$ les tangentes al et bl qui se coupent en $\mathrm{l}$; on demande la tangente au lieu décrit par le point $\mathrm{l}$ lorsque la transversale tourne autour du point $\mathrm{f}$.

Pour résoudre cette question, on remplace en $a$ et en $b$ les courbes $\mathbf{A}$ en $\mathbf{B}$ par des coniques osculatrices, ayant pour foyer commun le point $f$, et l'on cherche pour celles-ci l'axe d'homologie qui passe par le point $l$.

Cette droitc, qui est la tangente cherchée, passant aussi par le point de rencontre des directrices de ces conirques, est facile à déterminer.

Constructions du centre de courbure de la courbe, lieu des points dont les distances à deux courbes données sont dans un rapport constant.

Soient B, C (fig. $2^{\mathrm{a}}$.) les courbes données, A la ligne dont on vent construire le centre de courbure correspondant a un point quelconque $a$. L'on a $\frac{a b}{a c}=$ constante, et il est facile de voir que d'après cetle condition les tangentes al, $b l, c l$ aux trois courbes, A, B, C se coupent au mème point $l$.

Soient $o$ le point cherché, $f$ le centre de courbure de $\mathbf{B}$ en $b, f^{\prime}$ le centre de courbure de $\mathrm{C}$ en $c$; d'après le lemme précédent, on construit la tangente $\mathrm{L}$ au lieu décrit par le point $l$, considéré comme point de rencontre des tangentes $a l, b l$, de la manière suivante :

Du point $o$, on abaisse sur af la perpendiculaire op, et du point $p$ sur ao la perpendiculaire $p q$, la ligne $f q$ est perpendiculaire à la tangente cherchéc.

En considèrant le point $l$ comme le point de rencontre des tangentes al, $c l$ nous pouvons, d'après la réciproque du lemme, revenir de la connaissance de $\mathrm{L}$ à la détermination du centre de courbure $f^{\prime}$ de $\mathrm{C}$ par la construction suivante : du point $o$, on abaisse sur $a c$ la perpendiculaire $o p^{\prime}$; du point $p^{\prime}$, on abaisse sur ao la perpendieulaire $p^{\prime} q^{\prime}$; en menant la ligne $q^{\prime} f^{\prime}$ parallèlement à $q f$, on a une ligne perpendiculaire ì L, qui passe par le centre de courbure $f^{\prime}$ cherché. Nous avons ainsi une construction, que nous appellons construction (1), qui est symétrique par rapport aux points $f$ et $f^{\prime}$, et qui permet de déterminer l'un de ces points lorsque l'autre est donné 
ainsi que le point $o$; il nous reste à transformer cette construction afin de déterminer $o$ lorsque l'on donne $f$ et $f^{\prime}$.

Au point $f$, (figr. 2.), élevons sur af la perpendiculaire $f r$; au point $r$, élevons sur ao la perpendiculaire $r s$; la ligne so est parallèle à $f q$ : en effet, l'hexagone opqfirs inscrit dans l'angle oaf a dleja les cotés op, fr paralleles entre enx, ainsi que $p q, r s$; done os est parallèle à $f q$. On démontre de mème, en effectuant une constrtiction analogue pour $f^{\prime}$, que os' est parallèle à $f^{\prime \prime} q^{\prime}$; mais $f q$ et $f^{\prime \prime} q$ ' sont des lignes parallèles entre elles; done les points $s, o, s^{\prime}$ sont en ligne droite.

Nous avons done, pour déterminer le point $o$, la construction suivante, que nous appellerons, construction (2): aux points $f$ et $f^{\prime}$, on élive aux droites af el $a f^{\prime \prime}$ les perpendiculaires $f r, f^{\prime} r^{\prime}$; des points $r, r^{\prime}$, on éleve sur ha normale $a o$, qui est connue puisfu'on sait construire al, les perpendiculaires $r s, r s^{\prime}$; hat ligne $s s^{\prime}$ coupe lit normale ao au point ó cherché.

Celte construction symétrique par rapport aux points $f$ el $l^{\prime}$ peut se transformer de la manière suivante : prolongeons (fig. 2) rf jusqu'it sa renenutre $l^{\prime}$ avec al; les triangles l'fa et r'os' sont tels que leurs côtés se coupent en trois points en ligne droite : l' $f$ et $r^{\prime} o$ se coupent en $r$, af et s'o se coupent en $s$, $l^{\prime} a$ et $r^{\prime} s^{\prime}$ sont parallèles à la ligne $s r$; done ils ont leurs sommets sur trois droites concourantes, ainsi les lignes $l{ }^{\prime} r^{\prime}$, fo se coupent en $n$ sur $a f^{\prime}$. De là une construction, que nous appellerons, construction (3), qui lie les trois points $f, o, f$, el qui permet de déterminer o ou $f^{\prime}$ connaissant $f$ et l'un de ces points. Il existe une construction analogue qui permet de déterminer $o$ ou $f$ connaissant $f^{4}$ et l'un de ces points.

Applications. Lorsque les lignes B, C sont des circonférences, et que le rapport $\frac{a b}{a c}$ diffère de l'unité, la ligne $\mathrm{A}$ est une ovale de Descartes; lorsque le rapport constant est l'unité, $\mathbf{A}$ est une conique à centro; lorsque les lignes $\mathrm{B}$ et $\mathrm{C}$ se réduisent à un point et à une droite, $\mathbf{A}$ est une conique quelconque. Dans tous ces cas il est facile de construire le centre de courbure de $\mathrm{A}$.

En appliquant aux coniques les constructions données plus hant, on ne retroure pas la construction que nous avons employée dans la $2^{\text {eme }}$ solution de notre lemme, et qui nous a conduit à la première construction de $\mathbf{L}$.

Nous allons y arriver, comme cas particulier d'une nouvclle construction générale. Nous avous vu, (fig. 2) que si du point $o$ on abaisse sur af, af' les perpendiculaires $o p, o p^{\prime}$, et que si des points $p, p^{\prime}$ on abaisse sur ao les perpendiculaires $p q, p^{\prime} q^{\prime}$, les lignes $f q, f^{\prime} q^{\prime}$ sont paralléles. On peut opérer inversement: menons des points $f, f^{\prime}$ des parallèles quelconques qui rencontrent ao en deux points; en ces points, élevons sur ao des perpendiculaires qui coupent $a f$, $a f^{\prime}$; enfin de ces points d'intersection, élevons sur af, $a f^{t}$ des perpendiculaires qui se coupent en 
$\omega\left(^{*}\right)$; à chaque direction des parallèles issues des point $f, f^{\prime}$ correspond un point tel que $\omega$; il est facile de voir que tous ces points sont en ligne droite. Le centre de courbure cherehé est donc l'intersection de $a o$ et de cette droite. Pour construire celle-ci, nous allons chercher deux de ses points: si les parallèles menées des points $f, f^{\prime}$ sont perpendiculaires à $a 0$, on obtient un point $v$ de cetle droite en prolongeant jusqu'à leur rencontre les lignes $r f, r^{\prime} f^{\prime}$; si les parallèles menées des points $f, f^{\prime}$ se confondent en une seule droite $f f^{\prime}$, on obtient le point $u$ comme l'indique la figure et la ligne $u v$ coupe la normale ao au centre de courbure cherché.

Dans le cas particulier où l'on considère une conique, le point $\omega$ est sur la normale ao; donc e'est le centre de courbure de la conique. Telle est la construction que nous nous étions proposé de retrouver.

Application au problême de la caustique par réfraction. La ligne A, (fig. 2), est telle que pour un point quelconque $a$, on a $\frac{a b}{a c}=$ constante; mais $a b=a l$ sin. $b l a$ $=a l \sin . b a r, a c=a l \sin . c l a=a l \sin . c a r, \mathrm{~d} n \mathrm{nc} \frac{\sin . b a r}{\sin . c a r}=\frac{a b}{a c}=$ const. D'après cela, si $f a$ est la direction d'un rayon lumineux, A la ligne séparatrice des milieux, af enveloppe une caustique par réfraction dont $\mathrm{C}$ est la caustique secondaire.

En employant l'une quelconque des constructions précédentes, il est facile de déterminer le point $f^{\prime}$ où le rayon réfracté $a f^{\prime}$ touche son enveloppe.

Nous allons chercher maintenant la formule qui lie entre elles les longueurs $a f$, af', ao et les lignes trigonométriques des angles d'incidence el de réfraction. Pour cela nous ferons usage du théorème suivant:

Etant donnés un angle dont le sommet est $\mathrm{m}$ et un point quelconque o dans son plan, on a, en observant la règle des signes, quelle que soit la direction d'une droite passant par ce point et coupant les côtés de l'angle aux points $\mathrm{c}$ et $\mathrm{d}$.

$$
\left(\frac{1}{o d}-\frac{1}{o c}\right) \frac{1}{\sin . d o m}=\text { constante. }
$$

(Tansf. des prop. métriques des figures. Page 3).

Employons les lignes de la construction (3): soient $g$ le point où $f n$ coupe al', $i$ l'angle d'incidence el $i$ l'angle de réfraction; l'on a dans l'angle $r f n$ coupé par les transversales ro, $a l^{\prime}$ :

$$
\left(\frac{1}{a o}-\frac{1}{a r}\right) \frac{1}{\sin . i}=\left(\frac{1}{a g}-\frac{1}{a l}\right) \frac{1}{\sin . l a f o u \cos . i},
$$

l'angle l'nf coupé par les mêmes transversales donne:

(*) Ce point n'est pas sur la figure. 


$$
\left(\frac{1}{a o}-\frac{1}{a r^{\prime}}\right) \frac{1}{\sin . i^{i}}=\left(\frac{1}{a g}-\frac{1}{a l^{\prime}}\right) \frac{1}{\cos \cdot i^{i}},
$$

Divisant membre à membre ces deux égalités et remplaçant ar et ar' par leurs váleurs $\frac{a s}{\cos . i}, \frac{a s^{\prime}}{\cos . i^{i}}$, on a la relation cherchée.

Autrement. Employons les lignes de la construction (2): Soit $h$ le point où $s s^{\prime}$ coupe $a l$, l'on a dans l'angle rso coupé par les transversales ar, al:

$$
\left(\frac{1}{a 0}-\frac{1}{a r}\right) \frac{1}{\sin . i}=\frac{1}{a h \cos . i},
$$

dans l'angle $r^{\prime}$ 'so coupé par les mêmes transversales, on a :

$$
\left(\frac{1}{a 0}-\frac{1}{a r^{\prime}}\right) \frac{1}{\sin \cdot i^{i}}=\frac{1}{a h} \frac{1}{\cos \cdot i^{i}},
$$

en divisant membre à membre ces deux égalités, on en déduit la relation cherchée.

Remarque. Les constructions (1), (2), (3) ont été déduites de la première construction de $\mathbf{L}$; on peut $\mathrm{y}$ arriver en partant de la deuxième construction de cette droite.

Chatellerault Aoùt 1858. 